DOI: 10.15690/vsp.v16i4.1775

\title{
О.А. Жданова
}

Воронежский государственный медицинский университет им. Н.Н. Бурденко, Воронеж, Российская Федерация

\section{Глюкокортикостероидная терапия и физическое развитие детей со стероидчувствительным нефротическим синдромом: результаты ретроспективного исследования}

Контактная информация:

Жданова Ольга Александровна, кандидат медицинских наук, доцент кафедры клинической фармакологии ВГмУ им. Н.Н. Бурденко Адрес: 394030, Воронеж, ул. Студенческая, д. 10, тел.: +7 (473) 265-64-42, e-mail: olga.vr9@yandex.ru Статья поступила: 06.06.2017 г., принята к печати: 28.08.2017 г.

Увеличение массы тела и задержка роста - частые побочные эффекты длительной глюкокортикостероидной терапии у детей. Сроки возникновения и устранения глюкокортикостероидного ожирения, а также нарушений роста требуют дальнейшего исследования. Цель исследования: изучить связь глюкокортикостероидной терапии с изменением показателей физического развития детей со стероидчувствительным нефротическим синдромом (СЧНС). Методы. Проведено ретроспективное исследование историй болезни пациентов с СЧНС, госпитализированных в 2011-2014 гг. Лечение детей проводили в соответствии с Федеральными клиническими рекомендациями. Определяли Z-score (ANTHRO Plus) длины тела (рост), массы тела, индекса массы тела и корреляцию показателей физического развития с кумулятивной дозой и длительностью глюкокортикостероидной терапии. Результаты. Проанализированы данные о лечении 31 ребенка, из них 18 получали глюкокортикостероиды в течение 6 мес (группа 1), 13 - не получали глюкокортикостероиды $\geqslant 6$ мес (группа 2). Показатель Z-score массы тела у детей в указанных группах составил 1,64 \pm 1,54 и 0,05 \pm 1,19 ( $p=0,004)$, показатель Z-score индекса массы тела - 1,85 $\pm 1,64$ и -0,54 $\pm 1,14$ соответственно ( $p<0,001)$. Избыток массы тела и ожирение имелись только у детей группы 1 (у 6 и 9 соответственно). Z-score длины тела пациентов групп 1 и 2 были сопоставимы и не отличались от нормальных величин $(0,34 \pm 1,08$ и 0,52 $\pm 1,12$ соответственно; $p=0,655)$. Отмечена корреляция значений Z-score длины тела и кумулятивных доз глюкокортикостероидов ( $r=-0,87 ; p<0,001)$. Заключение. Длительный (не менее 6 мес) прием глюкокортикостероидов связан с развитием избыточной массы тела и ожирения у большинства детей с СЧнС. У пациентов, не использовавших гормональные препараты в течение $\geqslant 6$ мес, зафиксированы нормальные значения массы тела. Рост детей с СЧНС находился в диапазоне нормальных значений.

Ключевые слова: дети, нефротический синдром, глюкокортикостероиды, длина тела, масса тела, ожирение.

(Для цитирования: Жданова О.А. Глюкокортикостероидная терапия и физическое развитие детей со стероидчувствительным нефротическим синдромом: результаты ретроспективного исследования. Вопросы современной педиатрии. 2017; 16 (4): 291-296. doi: 10.15690/vsp.v16i4.1775)

Olga A. Zhdanova

Voronezh State Medical University named after N.N. Burdenko, Voronezh, Russian Federation

\section{Glucocorticosteroid Therapy and Physical Development of Children with Steroid-Sensitive Nephrotic Syndrome: a Retrospective Study}

Background. Body weight gain and growth retardation are common side effects of prolonged glucocorticosteroid therapy in children. Time for the appearance and elimination of glucocorticosteroid obesity as well as growth disorders require further investigations. Objective. Our aim was to study the relationship between glucocorticosteroid therapy and changes in physical development indices of children with steroid-sensitive nephrotic syndrome (SSNS). Methods. We carried out a retrospective study of case records of patients with SSNS hospitalized in 2011-2014. Treatment of children was carried out in accordance with the Federal Clinical Guidelines. The Z-score (ANTHRO Plus) was determined for body length (height), body weight, body mass index and correlation of physical development indices with a cumulative dose and duration of glucocorticosteroid therapy. Results. We analyzed data on the treatment of 31 children, 18 of them received glucocorticosteroids for 6 months (Group 1), 13 of them did not receive glucocorticosteroids $\geqslant 6$ months (Group 2). The Z-score of body weight in children in these groups was $1.64 \pm 1.54$ and $0.05 \pm 1.19(p=0.004)$, Z-score of body mass index was $1.85 \pm 1.64$ and $-0.54 \pm 1.14$, respectively $(p<0.001)$. Excess body weight and obesity were only observed in children of Group 1 (in 6 and 9, respectively). The Z-score of the body length of patients in groups 1 and 2 were comparable and did not differ from normal values $(0.34 \pm 1.08$ and $0.52 \pm 1.12$, respectively, $p=0.655)$. Correlation of $Z$-score values of the body length and cumulative doses of glucocorticosteroids was noted $(r=-0.87, p<0.001)$. Conclusion. Long-term (at least 6 months) glucocorticosteroid intake is associated with the development of overweight and obesity in most children with SSNS. In patients who did not use hormonal drugs for $\geqslant 6$ months, normal body weight values were recorded. The height of children with SSNS was within the range of normal values. Key words: children, nephrotic syndrome, glucocorticosteroids, body length, body weight, obesity.

(For citation: Zhdanova Olga A. Glucocorticosteroid Therapy and Physical Development of Children with Steroid-Sensitive Nephrotic Syndrome: a Retrospective Study. Voprosy sovremennoi pediatrii - Current Pediatrics. 2017; 16 (4): 291-296. doi: 10.15690/ vsp.v16i4.1775) 


\section{ОБОСНОВАНИЕ}

Нефротический синдром у детей характеризуется протеинурией > 3,0 г/сут (> 50 мг/кг массы тела в сутки или $>40 \mathrm{мг} / \mathrm{M}^{2}$ поверхности тела в час), снижением концентрации альбумина крови < 25 г/л, гиперлипидемией и развитием отеков $[1,2]$. Глюкокортикостероиды (ГКС) являются основными препаратами выбора в лечении стероидчувствительного нефротического синдрома (СЧНС) у детей. До использования ГКС и антибиотиков около $40 \%$ из них умирали [3]. У 80-90\% пациентов при приеме ГКС наступает ремиссия заболевания [1]. В зависимости от ответа на терапию ГКС выделяют СЧНС и стероидрезистентный нефротический синдром (СРНС) [2].

Согласно международным и российским рекомендациям, преднизолон для лечения нефротического синдро-

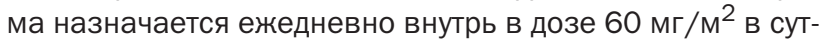
ки, или 2 мг/кг в сутки (не более 60 мг/сут), непрерывно, в течение 4-6 нед с последующим переходом на прием препарата через день в дозе 40 мг/м² , или 1,5 мг/кг (максимум 40 мг через день) в течение 4-6 нед с постепенным снижением дозы и отменой препарата [2-4]. Общая длительность терапии ГКС составляет 4-5 мес [2]. Прием препарата через день (альтернирующий режим) предложен для поддержания ремиссии в связи с тем, что при таком приеме лечение оказывает меньшее негативное влияние на рост ребенка [5]. Вместе с тем альтернирующий режим приема ГКС увеличивает продолжительность ремиссии по сравнению с интермиттирующим (прерывистым) режимом приема [3].

Дети с часто рецидивирующим нефротическим синдромом (ЧРНС) и стероидзависимым нефротическим синдромом (СЗНС) - клиническими вариантами СЧНС - нуждаются в длительной терапии ГКС, которая сопряжена с развитием серьезных побочных эффектов, включая задержку роста и ожирение [3]. Развитие ожирения при приеме ГКС является общеизвестным побочным эффектом, но лишь в небольшом числе исследований изучались сроки возникновения и обратного развития ГКС-ожирения у детей $[6,7]$. По разным данным, частота развития ожирения составляет 35-43\% [7]. Уменьшение дозы ГКС приводит к уменьшению массы тела, но не у всех пациентов она приходит к норме по окончании терапии [8]. B. J. Foster и соавт. показали, что как недавний, так и отдаленный прием ГКС ассоциировался с высоким риском развития ожирения у детей с нефротическим синдромом [6].

Целью нашего исследования было изучить связь терапии ГКС с показателями физического развития детей со стероидчувствительным нефротическим синдромом.

\section{МЕТОДЫ}

\section{Дизайн исследования}

Проведено ретроспективное исследование по схеме «случай-контроль».

\section{Критерии соответствия}

Критерии включения:

- дети с СЧнС в возрасте от 3 до 18 лет;

- проведение ребенку стандартной стероидной терапии в дебюте и при рецидивах заболевания;

- наличие в истории болезни подписанного родителями или законными представителями пациента информированного согласия на обработку данных и их использования в научных целях.

Критерии невключения:

- дети с врожденным нефротическим синдромом, с нефротическим синдромом, протекающим на фоне тяже- лой соматической, неврологической или генетической патологии, со вторичным нефротическим синдромом;

- пациенты с нефротическим синдромом, лечившиеся в период исследования по другим схемам, кроме стандартной стероидной терапии (пульс-терапия метилпреднизолоном, прием цитостатиков).

\section{Диагностические критерии}

Диагноз нефротического синдрома устанавливали при наличии протеинурии $>40 \mathrm{мг} / \mathrm{M}^{2}$ в час, гипоальбуминемии < 25 г/мл или отеков и гиперлипидемии [2, 3]. СЧНС определяли при достижении пациентами полной ремиссии на фоне терапии преднизолоном в дозе 2 мг/кг в сутки, или 60 мг/м² в сутки, в течение 4 нед [2, 3]. ЧРНС диагностировали, когда рецидивы заболевания возникали в течение $6 \mathrm{мес} \geqslant 2$ раз. СЗНС отмечали у детей при возникновении рецидива в период снижения дозы преднизолона или не позднее чем через 2 нед после отмены препарата [2, 3].

\section{Протокол лечения}

Лечение проводилось в соответствии с протоколом Международной группы по заболеваниям почек у детей (International Study of Kidney Disease in Children, ISKDC) и Федеральными клиническими рекомендациями [2, 3]. В дебюте заболевания преднизолон назначали в дозе 60 мг/м² в сутки ежедневно в 3 приема в течение 6 нед, затем - 40 мг/м² в 48 ч в 1 прием в альтернирующем режиме в течение 4-6 нед с последующим снижением дозы на 5-10 мг в неделю до полной отмены [2, 3]. При рецидиве заболевания применяли преднизолон в дозе

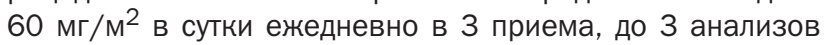
мочи без протеинурии (отношение белка к креатинину мочи < 20 мг/ммоль или наличие белка < 1+ при определении тест-полосками), затем продолжали терапию преднизолоном в альтернирующем режиме в дозе 40 мг/м² в 48 ч в 1 прием в течение 4-6 нед с последующим снижением дозы на 5-10 мг в неделю до полной отмены [2, 3]. Лечение осложнений ГКС-терапии осуществляли в соответствии с клиническими рекомендациями [2, 3].

\section{Источники данных}

Анализировали медицинскую документацию (истории болезни) детей с СЧНС, соответствующих критериям включения, госпитализированных в нефрологическое отделение Воронежской областной детской клинической больницы № 1 в период с 2011 по 2014 г.

\section{Оценка физического развития детей}

В числе показателей физического развития учитывали рост и массу тела, а также индекс массы тела (ИМТ). Оценку физического развития производили при поступлении ребенка в нефрологическое отделение, далее еженедельно. В случаях наличия отеков учитывалась масса тела ребенка, измеренная после купирования отечного синдрома.

Для сравнительной оценки значений роста и массы тела детей разного пола и возраста вычисляли Z-score с использованием программы WHO ANTHRO Plus [9]. При этом исходили из того, что ИМТ является более информативным показателем для оценки недостаточности или избыточности питания у детей и подростков, чем масса тела; стандартные нормативы массы тела предложены экспертами Всемирной организации здравоохранения (ВОЗ) только для детей в возрасте до 10 лет [9, 10]. Значения Z-score ИМТ для возраста (body-mass-index-for-age z-score, BAZ) рассчитывали с использованием стандартных нормативов В0З согласно Федеральным клиническим рекомендациям по лечению детей с эндокринными заболеваниями 
[11]. K нормальным значениям BAZ относили показатели, находящиеся в диапазоне от -1 до +1 , легкая недостаточность питания включала диапазон значений от -1 до -2 , недостаточность питания или истощение определяли при величинах < -2, избыток массы тела - при величинах от +1 до +2 , ожирение $-\geqslant+2[9,11]$. При анализе Z-score длины тела (роста) для возраста (height-for-age z-score, HAZ) к возрастной норме относили значения в диапазоне от -2 до +2, низкорослость диагностировали при значениях $\mathrm{HAZ}<-2$, высокий рост $->+2$.

Для сравнительного анализа показателей физического развития у детей, получавших и не получавших ГКС в течение 6 мес до госпитализации, использовали данные одной госпитализации для каждого ребенка: на фоне приема максимальной дозы ГКС (группа 1) и в период наиболее длительной ремиссии заболевания (группа 2).

\section{Исходы исследования}

Основной исход исследования: отклонение показателей физического развития детей (рост, масса тела, ИМТ) от стандартов ВОЗ у детей с СЧНС в период проведения терапии ГКС и после ее окончания.

Дополнительные исходы исследования: связь между отклонениями показателей физического развития и полученной ребенком дозой ГКС.

\section{Этическая экспертиза}

Проведение исследования одобрено Этическим комитетом Воронежской государственной медицинской академии им. Н. Н. Бурденко (протокол № 6 от 19.10.2013 г.). Во всех историях болезни детей имелось информированное согласие на обработку данных, в т. ч. для проведения научных исследований.

\section{Статистический анализ}

Размер выборки предварительно не рассчитывался. Анализ данных проведен с использованием пакета статистических программ STATISTICA v. 6.0. (StatSoft Inc., США). Количественные показатели, имеющие нормальное распределение, описаны с указанием среднего арифметического значения и стандартного отклонения. Сравнение количественных показателей в группах выполнено с применением t-теста Стьюдента для независимых выборок. При отклонении гипотезы о нормальном распределении исследуемых показателей для анализа различий в двух группах использовали непараметрический критерий Манна-Уитни (U). Такие показатели описаны с указанием медианы (25-го; 75-го процентилей). Для сравнения качественных показателей использовали точный критерий Фишера (учитывая малый размер выборок). Для выявления связи между показателями физического развития и полученной дозой ГКС применяли корреляционный анализ - рассчитывали коэффициент ранговой корреляции Спирмена.

\section{РЕЗУЛЬТАТЬ}

\section{Характеристика выборки}

В исследовании проанализированы данные 31 пациента со СЧНС, из них 18 детей получали ГКС в течение 6 мес до госпитализации в стационар (группа 1), 13 не получали (группа 2). В группе 1 чРНС был у 4 больных, СЗНС - у 3 человек. Группы различались по возрасту пациентов и длительности заболевания (табл. 1). Дети группы 2 поступали для планового обследования в период ремиссии заболевания. Большинство детей поступали в стационар неоднократно.

\section{Основные результаты исследования}

Оценка физического развития детей с СЧНС с использованием программы WHO ANTHRO Plus показала отличия полученных Z-score массы тела и ИМТ в группе 1, получавших ГКС в течение предыдущих месяцев, от стандартных показателей (представленных нулевым средним значением и стандартным отклонением равным 1) и Z-score детей группы 2, не получавших ГКС 6 мес и более (табл. 2). По величине Z-score длины тела группы детей с СЧНС, получавшие и не получавшие ГКС в течение 6 мес до госпитализации, не различались.

Частота встречаемости значений Z-score длины тела и ИМТ, находящихся в пределах возрастной нормы и выходящих за пределы средних величин у детей с СЧНС, представлена в табл. 3. Распределение Z-score длины тела детей групп 1 и 2, получавших и не получавших ГКС в течение

Таблица 1. Сравнительная характеристика детей со стероидчувствительным нефротическим синдромом, получавших и не получавших глюкокортикостероиды в течение 6 мес до госпитализации

Table 1. Comparative analysis of children with steroid-sensitive nephrotic syndrome who received and did not receive glucocorticosteroids for 6 months before admission

\begin{tabular}{|c|c|c|c|}
\hline Показатели & Группа $1(n=18)$ & Группа $2(n=13)$ & $p$ \\
\hline $\begin{array}{l}\text { Возраст, годы } \\
\min -\max \end{array}$ & $\begin{array}{c}5,6(5,0 ; 8,0) \\
4-14\end{array}$ & $\begin{array}{c}9,1(7,0 ; 12,9) \\
3-18\end{array}$ & 0,032 \\
\hline Девочки, абс. (\%) & $10(56)$ & $5(39)$ & 0,348 \\
\hline $\begin{array}{l}\text { Место проживания, абс. (\%): } \\
\text { - город } \\
\text { - село }\end{array}$ & $\begin{array}{c}7(39) \\
11(61)\end{array}$ & $\begin{array}{l}6(46) \\
7(54)\end{array}$ & 0,686 \\
\hline $\begin{array}{l}\text { Длительность заболевания, мес } \\
\text { min-max }\end{array}$ & $\begin{array}{c}22(11 ; 38) \\
4 \text { мес-11 лет }\end{array}$ & $\begin{array}{c}63(27 ; 122) \\
11 \text { мес-16 лет }\end{array}$ & 0,009 \\
\hline $\begin{array}{l}\text { Возраст дебюта заболевания, мес } \\
\min -\max \end{array}$ & $\begin{array}{l}45(33 ; 61) \\
1,8-8,4 \text { лет }\end{array}$ & $\begin{array}{c}36(29 ; 66) \\
9 \text { мес-8 лет }\end{array}$ & 0,346 \\
\hline $\begin{array}{l}\text { Госпитализации, абс. } \\
\text { • обострение, абс. (\%) } \\
\text { • дебют, абс. (\%) }\end{array}$ & $\begin{array}{c}71 \\
35(49) \\
9(13)\end{array}$ & $\begin{array}{c}16 \\
2(13) \\
-\end{array}$ & 0,001 \\
\hline $\begin{array}{l}\text { Длительность ремиссии, мес } \\
\min -\max \end{array}$ & $\begin{array}{c}2(2 ; 4) \\
1-10 \text { мес }\end{array}$ & $\begin{array}{c}38(20 ; 108) \\
11 \text { мес-13 лет }\end{array}$ & 0,001 \\
\hline
\end{tabular}

Примечание. Группа 1 - на фоне приема максимальной дозы ГКс, группа 2 - в период наиболее длительной ремиссии заболевания. Note. Group 1 - while taking the maximum dose of glucocorticosteroids, Group 2 - in the period of the longest remission of the disease. 
6 мес до госпитализации, не различалось. Вместе с тем у детей, лечившихся ГКС, отмечен сдвиг значений Z-score массы тела и ИМТ в сторону избытка массы тела и ожирения (см. табл. 3). Среди пациентов группы 2, не получавших ГКС в течение 6 мес и более перед госпитализацией, не было выявлено детей с избытком массы тела и ожирения. У части пациентов отмечался недостаток массы тела (см. табл. 3).
Дети группы 1 получили за весь период болезни более высокую кумулятивную дозу ГКС по сравнению с детьми группы 2 (табл. 4). У них отмечалась и большая длительность приема ГКС. В указанную группу вошли пациенты с ЧРНС и СЗНС, получившие больше курсов ГКС по сравнению с детьми с нечасто рецидивирующим нефротическим синдромом.

Таблица 2. Средние значения Z-score показателей физического развития детей с нефротическим синдромом Table 2. Mean values of Z-score indices of physical development of children with nephrotic syndrome

\begin{tabular}{|l|c|c|c|}
\hline \multicolumn{1}{|c|}{ Показатели } & Группа 1 $(\boldsymbol{n = 1 8 )}$ & Группа 2 (n= 13) & 0,52 $\pm 1,12$ \\
\hline Z-score длины тела (роста) & $0,34 \pm 1,08$ & 0,655 \\
\hline Z-score массы тела & $1,64 \pm 1,54$ & $0,05 \pm 1,19$ & $-0,54 \pm 1,14$ \\
\hline Z-score ИМТ & $1,85 \pm 1,64$ & 0,000 \\
\hline
\end{tabular}

Примечание. ИМТ - индекс массы тела.

Note. ИМТ — body mass index.

Таблица 3. Частота встречаемости Z-score показателей физического развития детей со стероидчувствительным нефротическим синдромом

Table 3. Incidence of Z-score indices of physical development of children with steroid-sensitive nephrotic syndrome

\begin{tabular}{|c|c|c|c|}
\hline Показатели* & Группа 1, абс. (\%) & Группа 2, абс. (\%) & $p^{\star *}$ \\
\hline Z-score длины тела (роста) & $n=18$ & $n=13$ & - \\
\hline $\begin{array}{l}\text { Низкий } \\
\text { Ниже среднего } \\
\text { Средний } \\
\text { Выше среднего } \\
\text { Высокий }\end{array}$ & $\begin{array}{c}0 \\
3(17) \\
10(55) \\
5(28) \\
0\end{array}$ & $\begin{array}{c}0 \\
2(15) \\
7(54) \\
3(23) \\
1(8)\end{array}$ & $\begin{array}{l}- \\
0,690 \\
0,786 \\
0,904 \\
0,898\end{array}$ \\
\hline Z-score массы тела*** & $n=13$ & $n=7$ & - \\
\hline $\begin{array}{l}\text { Низкий } \\
\text { Ниже среднего } \\
\text { Средний } \\
\text { Выше среднего } \\
\text { Высокий }\end{array}$ & $\begin{array}{c}0 \\
0 \\
5(38,5) \\
3(23) \\
5(38,5)\end{array}$ & $\begin{array}{c}1(14) \\
0 \\
6(86) \\
0 \\
0\end{array}$ & $\begin{array}{l}0,747 \\
- \\
0,120 \\
0,470 \\
0,176\end{array}$ \\
\hline Z-score ИМТ & $n=14$ & $n=13$ & - \\
\hline $\begin{array}{l}\text { Низкий } \\
\text { Ниже среднего } \\
\text { Средний } \\
\text { Выше среднего (избыток массы тела) } \\
\text { Высокий (ожирение) }\end{array}$ & $\begin{array}{c}0 \\
0 \\
4(28,5) \\
4(28,5) \\
6(43)\end{array}$ & $\begin{array}{l}2(15) \\
2(15) \\
9(70) \\
0 \\
0\end{array}$ & $\begin{array}{l}0,430 \\
0,430 \\
0,084 \\
0,122 \\
0,027\end{array}$ \\
\hline
\end{tabular}

Примечание. Группа 1 - на фоне приема максимальной дозы ГКС, группа 2 - в период наиболее длительной ремиссии заболевания. * - коридоры значений Z-score: низкий $-<-2$, ниже среднего - от - 2 до -1 , средний - от -1 до +1, выше среднего - от 1 до 2 , высокий $->2$. ** - рассчитано по критерию $\chi^{2}$ с поправкой Йетса для таблиц сопряженности $2 \times 2$; значение величины $p$ для всей таблицы $5 \times 2(\mathrm{df}=4)$ составило для длины тела 0,833, для массы тела $-0,091$, для ИМТ $-0,004 . * * *-$ значения массы тела в программе ANTHRO Plus представлены только для детей в возрасте до 10 лет; у детей с отеками значения массы тела и ИМт в программе ANTHRO Plus не учитываются.

Note. Group 1 - while taking the maximum dose of glucocorticosteroids, Group 2 - in the period of the longest remission of the disease. * Ranges for Z-score values: low $-<-2$, below the average - from -2 to -1 , average - from -1 to +1 , above the average - from 1 to 2 , high $->2$. ${ }^{* *}$ Calculated by $\chi^{2}$ criterion with Yates' correction for contingency tables $2 \times 2$; the value of $p$ for the entire table $5 \times 2(\mathrm{df}=4)$ was 0.833 for the body length, 0.091 for the body weight, 0.004 for the BMI. *** Body weight values in the ANTHRO Plus program are given only for children under 10 years; in children with edema, body weight and BMI values in the ANTHRO Plus program are not considered.

Таблица 4. Глюкокортикостероидная терапия детей со стероидчувствительным нефротическим синдромом Table 4. Glucocorticosteroid therapy of children with steroid-sensitive nephrotic syndrome

\begin{tabular}{|l|c|c|c|}
\hline \multicolumn{1}{|c|}{ Показатели } & Группа 1 (n= 18) & Группа 2 (n=13) & p \\
\hline Кумулятивная доза ГКС (за весь период болезни), мг/кГ & $240(199 ; 315)$ & $145(102 ; 181)$ & 0,001 \\
\hline Длительность приема ГКС, сут & $485(221 ; 660)$ & $300(180 ; 365)$ & 0,051 \\
\hline
\end{tabular}

Примечание. ГКС - глюкокортикостероиды.

Note. ГКС - glucocorticosteroids. 
Таблица 5. Значения коэффициента ранговой корреляции Спирмена Z-score показателей физического развития и полученной дозы глюкокортикостероидов у детей с нефротическим синдромом

Table 5. Values of the Spearman's rank correlation coefficient of Z-score of physical development indices and the received dose of glucocorticosteroids in children with nephrotic syndrome

\begin{tabular}{|c|c|c|c|c|c|c|}
\hline Показатели & $\begin{array}{c}\text { Кумулятивная } \\
\text { доза (мг) }\end{array}$ & $\begin{array}{c}\text { Кумулятивная } \\
\text { доза (мг/кг) }\end{array}$ & $\begin{array}{c}\text { Доза за } 6 \text { мес } \\
\text { (мг) }\end{array}$ & $\begin{array}{c}\text { Доза за } 6 \text { мес } \\
\text { (мг/кг) }\end{array}$ & $\begin{array}{l}\text { Доза за } 6 \text { мес } \\
\text { (мг/кг в сутки) }\end{array}$ & $\begin{array}{c}\text { Длительность } \\
\text { приема ГКС }\end{array}$ \\
\hline \multicolumn{7}{|c|}{ Группа $1(n=18)$} \\
\hline Масса тела & $0,70 *$ & 0,51 & 0,37 & $-0,14$ & $-0,38$ & 0,54 \\
\hline Рост & 0,45 & 0,10 & $-0,04$ & $-0,38$ & $-0,42$ & 0,40 \\
\hline ИМТ & 0,22 & 0,09 & 0,33 & 0,06 & 0,13 & 0,04 \\
\hline \multicolumn{7}{|c|}{ Группа 1, дети с редкими рецидивами нефротического синдрома $(n=11)$} \\
\hline Масса тела & 0,57 & 0,22 & 0,43 & $-0,28$ & $-0,50$ & 0,48 \\
\hline Рост & $0,64 *$ & 0,21 & $-0,05$ & $-0,68^{*}$ & $-0,87 * *$ & $0,71 *$ \\
\hline ИМТ & 0,29 & 0,06 & 0,38 & 0,00 & $-0,22$ & 0,70 \\
\hline \multicolumn{7}{|c|}{ Группа 2 ( $(n=13)$} \\
\hline Масса тела & $-0,18$ & $-0,64$ & - & - & - & $-0,24$ \\
\hline Рост & $-0,02$ & $-0,42$ & - & - & - & $-0,07$ \\
\hline ИМТ & $-0,36$ & $-0,57$ & - & - & - & $-0,08$ \\
\hline
\end{tabular}

Примечание. ГКС - глюкокортикостероиды, ИМТ - индекс массы тела. $*-p<0,05, * *-p<0,001$.

Note. ГКС - glucocorticosteroids, ИМТ — body mass index. * $-p<0.05, * *-p<0.001$.

\section{Дополнительные результаты исследования}

У детей группы 1 отмечена положительная корреляция массы тела с общей кумулятивной дозой ГКС, роста с кумулятивной дозой и длительностью приема ГКС, отрицательная корреляция значений длины тела и дозы, полученной пациентом в течение последних 6 мес (табл. 5). Значимой является отрицательная корреляция между ростом и дозами ГКС (мг/кг и мг/кг в сутки), полученными пациентом в течение 6 мес ( $p=0,021$ и 0,000 соответственно). Увеличение получаемой дозы ГКС приводило к уменьшению значений длины тела. У пациентов группы 2, не получавших гормон в течение длительного времени, такой зависимости не получено.

\section{ОБСУЖДЕНИЕ}

\section{Резюме основного результата исследования}

Пациенты, получавшие ГКС в течение 6 мес до поступления в стационар, имели более высокие значения массы тела и ИМТ по сравнению с детьми, не получавшими ГКС в течение $\geqslant 6$ мес. У 2/3 пациентов, не получавших ГКС в течение 6 мес и более, ИМТ соответствовал норме, не было выявлено избытка массы тела и ожирения. Рост детей с нефротическим синдромом не отличался от величин, характерных для здоровых детей и подростков. Установлена тенденция к уменьшению значений длины тела (роста) у детей с СЧНС в периоды приема максимальных доз ГКС.

\section{Обсуждение основного результата исследования}

Увеличение массы тела детей в период приема ГКС описано во многих исследованиях [6-8]. Повышение аппетита и связанное с ним увеличение массы тела относятся к первым проявлениям побочного действия ГКС [13], наблюдаемым даже при применении их короткими (до 14 сут) курсами [14]. Это самый частый побочный эффект длительной терапии стероидными гормонами, обусловленный влиянием препаратов на обмен веществ $[12,13,15]$. Важным остается вопрос длительности существования ГКС-индуцированного ожирения у детей. По данным разных авторов, перенесенный в детстве СЧнС приводил к развитию избытка массы тела и ожи- рения у 8-23\% взрослых $[8,16]$. J. Foster и соавт. показали, что высокий риск ожирения связан как с недавним (в течение последних 6 мес до поступления в стационар), так и отдаленным приемом ГКС. Вместе с тем риск развития ожирения у пациентов, получавших преднизолон в последние 6 мес, был значительно выше (относительный риск составил 26,1 и 5,2 соответственно) [6]. В нашем исследовании у 83\% пациентов, получавших ГКС в течение предыдущих 6 мес, отмечены избыток массы тела и ожирение, тогда как среди детей, не получавших ГКС в течение 6 мес и более, пациентов с избытком массы тела и ожирением не выявлено. У детей, не имеющих частых рецидивов нефротического синдрома и стероидзависимости (группа 2), в период длительной ремиссии заболевания масса тела возвращалась к нормальным значениям. Отделенный прием ГКС у пациентов этой группы не оказал влияния на массу тела.

В большинстве рекомендаций по использованию ГКС указано, что их длительный прием может приводить к задержке роста детей $[6,15]$. ГКС тормозят синтез белка в элементах соединительной ткани и мышцах, нарушают образование белкового каркаса костей и отложение в них кальция [13]. Многими исследователями обсуждается вопрос о допустимых дозах ГКС, не препятствующих нормальному росту ребенка $[17,18]$. Изучалось влияние на рост постоянного, интермиттирующего и альтернирующего режимов терапии ГКС. Для поддержания ремиссии при нефротическом синдроме используется альтернирующий режим, поскольку он оказывает менее негативное влияние на рост ребенка [5].

В работе J. Simmonds и соавт. оценивался рост 41 ребенка в возрасте от 1,9 до 13,2 лет с СЗНС. Длительность наблюдения составила от 1,4 до 8,4 (в среднем 4,2) лет. Дети получали преднизолон в дозе от 0,06 до 1,45 мг/кг (в среднем 0,44) в сутки. Показано, что применение преднизолона в указанных дозах при лечении СЗНС у детей не оказывало существенного влияния на рост. Только в периоды применения доз более 0,75 мг/кг в сутки наблюдалось некоторое снижение роста [18].

В нашем исследовании рост детей двух групп (на фоне приема максимальной дозы ГКС и в период наиболее 
длительной ремиссии заболевания) не отличался от показателей, характерных для здоровых детей и подростков. Не было выявлено пациентов с задержкой роста. Однако рост детей, обследованных в период приема стандартной стероидной терапии, был ниже, чем у пациентов, не получающих ГКС. Стандартная стероидная терапия включает высокие (1-2 мг/кг в сутки) дозы ГКС, которые могут приводить к замедлению темпов роста ребенка в период приема. Установлена значимая отрицательная корреляция между приемом высоких доз ГКС и более низкими значениями длины тела детей с СЧНС.

\section{Ограничения исследования}

К ограничениям исследования относятся его ретроспективный характер и небольшой объем выборки. Проспективное исследование с оценкой антропометрических данных в одинаковые сроки у всех пациентов позволило бы более полно оценить влияние дозы ГКС на физическое развитие детей. Небольшое число пациентов с ЧРНС и СЗНС связано с включением в исследование детей, получавших только стероидную терапию внутрь и не получавших другие виды лечения (пульс-терапия, прием цитостатиков).

\section{ЗАКЛЮЧЕНИЕ}

Длительный (не менее 6 мес) прием ГКС сочетается с развитием избыточной массы тела и ожирения у большинства пациентов с СЧНС. Напротив, у большинства детей, не использовавших ГКС в течение $\geqslant 6$ мес, наблюдались нормальные значения массы тела, а также не зафиксировано избытка массы тела и ожирения. Рост детей с СЧНС находился в диапазоне нормальных значений. Обнаружена тенденция к уменьшению роста в периоды приема максимальных доз ГКС. Результаты исследования могут быть использованы для прогнозирования влияния ГКС на физическое развитие у пациентов с нефротическим синдромом, получающих разные курсы ГКС.

\section{ИСТОЧНИК ФИНАНСИРОВАНИЯ}

Не указан.

\section{FINANCING SOURCE}

Not specified.

\section{КОНФЛИКТ ИНТЕРЕСОВ}

Автор статьи подтвердил отсутствие конфликта интересов, о котором необходимо сообщить.

\section{CONFLICT OF INTERESTS}

Not declared.

\section{ORCID}

О. А. Жданова http:/orcid.org/0000-0002-3917-0395

\section{СПИСОК ЛИТЕРАТУРЫ}

1. Обухова В.А., Длин В.В. Факторы риска часто рецидивирующего течения стероид-чувствительного нефротического синдрома у детей // Российский вестник перинатологии и педиатрии. - 2014. - T. 59. - № 6 - C. 79-83. [Obukhova VA, Dlin VV. Risk factors of frequent relapses of steroid-sensitive nephrotic syndrome in children. Rossiiskii vestnik perinatologii $i$ pediatrii. 2014;59(6):79-83. (In Russ).]

2. Нефротический синдром у детей. Клинические рекомендации. [Nefroticheskii sindrom u detei. Klinicheskie rekomendatsii. (In Russ).] Доступно по: http://www.pediatr-russia.ru/sites/ default/files/file/kr_nefr.pdf. Ссылка активна на 02.06.2017.

3. Клинические практические рекомендации KDIGO по лечению гломерулонефритов. [KDIGO Clinical Practice Guideline for Glomerulonephritis. Kidney International supplements. (In Russ).] Доступно по: http://kdigo.org/clinical_practice_guidelines/pdf/KDIGO\%20GN\%20 Russian\%20Full\%20Text.pdf. Ссылка активна на 02.06.2017.

4. Lombel RM, Gipson DS, Hodson EM; Kidney Disease: Improving Global 0. Treatment of steroid-sensitive nephrotic syndrome: new guidelines from KDIGO. Pediatr Nephrol. 2013;28(3):415-426. doi: 10.1007/s00467-012-2310-x.

5. Broyer M, Guest G, Gagnadoux MF. Growth rate in children receiving alternate-day corticosteroid treatment after kidney transplantation. J Pediatr. 1992;120(5):721-725. doi: 10.1016/ s0022-3476(05)80234-3.

6. Foster BJ, Shults J, Zemel BS, Leonard MB. Risk factors for glucocorticoid-induced obesity in children with steroid-sensitive nephrotic syndrome. Pediatr Nephrol. 2006;21(7):973-980. doi: 10.1007/s00467-006-0100-z.

7. Hjorten R, Anwar Z, Reidy KJ. Long-term outcomes of childhood onset nephrotic syndrome. Front Pediatr. 2016;4:53. doi: 10.3389/ fped.2016.00053.

8. Ishikura K, Yoshikawa $\mathrm{N}$, Nakazato $\mathrm{H}$, et al. Morbidity in children with frequently relapsing nephrosis: 10-year follow-up of a randomized controlled trial. Pediatr Nephrol. 2015;30(3):459-468. doi: 10.1007/s00467-014-2955-8.

9. who.int [Internet]. WHO growth reference 5-19 years. Application tools. WHO AnthroPlus software [cited 2017 Jun 9]. Available from: http://www.who.int/growthref/tools/en/.

10. who.int [Internet]. WHO child growth standards: training course on child growth assessment. Geneva; WHO [cited 2017 Jun 9].
Available from: http://www.who.int/entity/childgrowth/training/ module_c_interpreting_indicators_ru.pdf?ua $=1$.

11. Федеральные клинические рекомендации (протоколы) по ведению детей с эндокринными заболеваниями / Под ред. Дедова И.И., Петерковой В.А. - М.: Практика; 2014. - С. 163182. [Federal'nye klinicheskie rekomendatsii (protokoly) po vedeniyu detei s endokrinnymi zabolevaniyami. Ed by Dedov I.I., Peterkova V.A. Moscow: Praktika; 2014. p. 163-182. (In Russ).]

12. Минакова О.В., Жданова О.А., Настаушева Т.Л. Современные изменения региональных справочных показателей длины и массы тела детей и подростков Воронежской области // Системный анализ и управление в биомедицинских системах. - 2017. T. 16. - № 1 - C. 110-118. [Minakova OV, Zhdanova OA, Nastausheva TL. Comparative description of physical development (height and weight) of children 14 years old in Voronezh Region. System analysis and management in biomedical systems. 2017; 16(1):110-118. (In Russ).]

13. Ландышев Ю.С. Механизмы действия и основные терапевтические эффекты глюкокортикоидов // Амурский медицинский журнал. - 2014. - № 1 - C. 10-29. [Landyshev YS. Mechanisms of action and therapeutic effects of basic glucocorticoids. Amurskii meditsinskii zhurnal. 2014;(1):10-29. (In Russ).]

14. Aljebab F, Choonara I, Conroy S. Systematic review of the toxicity of short-course oral corticosteroids in children. Arch Dis Child. 2016;101(4):365-370. doi: 10.1136/archdischild-2015309522.

15. Aljebab F, Choonara I, Conroy S. Long-course oral corticosteroid toxicity in children. Arch Dis Child. 2016;101(9):e2. doi: 10.1136/ archdischild-2016-311535.57.

16. Skrzypczyk P, Panczyk-Tomaszewska M, Roszkowska-Blaim M, et al. Long-term outcomes in idiopathic nephrotic syndrome: from childhood to adulthood. Clin Nephrol. 2014;81(3):166-173. doi: 10.5414/CN108044.

17. Hahn D, Hodson EM, Willis NS, Craig JC. Corticosteroid therapy for nephrotic syndrome in children. Cochrane Database Syst Rev. 2015;(3):CD001533. doi: 10.1002/14651858.CD001533.pub5.

18. Simmonds J, Grundy N, Trompeter R, Tullus K. Long-term steroid treatment and growth: a study in steroid-dependent nephrotic syndrome. Arch Dis Child. 2010;95(2):146-149. doi: 10.1136/ adc.2007.129957. 